\title{
Irene Caiazzo
}

\section{Animae sequuntur corpora}

\section{Le philosophe, les astres et la physiognomonie au XIII ${ }^{\mathrm{e}}$ siècle}

Depuis quelques décennies les études sur la figure et le rôle de l'intellectuel ou philosophe ou magister ou lettré et, par conséquent, sur la vie philosophique que l'on pouvait mener au Moyen Age se sont considérablement multipliées ; il serait certes impossible d'en rendre compte de manière adéquate ici`. L'historiographie philosophique se montre consensuelle sur le fait que ce sont les maîtres de la Faculté des Arts (en particulier de Paris) au XIII ${ }^{e}$ siècle qui s'interrogent les premiers sur le philosophe, la vie philosophique et la félicité intellectuelle, l'assimilation progressive de l'Ethique à Nicomaque et de ses commentaires étant le moteur principal. Sans doute faudrait-il nuancer cette vision trop « universitaire » et peut-être était-il possible d'être et de se conduire en philosophe ailleurs qu'à la Faculté des Arts. Sénèque, Cicéron ou encore le Boèce de la Consolation de Philosophie offraient des modèles de vie philosophique : ces auteurs étaient lus en dehors de l'université et cela même avant la naissance de cette dernière. Cependant, il faut bien admettre que le philosophe (ou le lettré) au XII ${ }^{\mathrm{e}}$ siècle est un individu, et que sa réputation ne tient qu'à lui seul en quelque sorte - que l'on songe à Abélard, Jean de Salisbury, Guillaume de Conches ou Adélard de Bath. En revanche, au XIII ${ }^{\mathrm{e}}$ siècle le groupe prend sans conteste le dessus ; l'émergence d'une nouvelle élite, la corporation des magistri artium, pousse ces derniers à une réflexion sur eux-mêmes et sur ce qui les détermine en tant que tels : bref, comment sélectionner ou à quoi reconnaît-on les membres de cette nouvelle catégorie socioprofessionnelle qui naît avec l'université occidentale, permettant un brin de mobilité et ascension sociale ? Les questions de la vie philosophique, de la noblesse et de la félicité intellectuelle sont en effet intimement liées : qui est le philosophe ? Pourquoi est-il philosophe ? D’autre part, dans un contexte nonuniversitaire, le notaire Brunetto Latini pose lui aussi la question de la « felicité » et de l'« home felix » dans le Livre dou Tresor rédigé en picard dans les années

\footnotetext{
*Achevé pendant l'été 2015, ce texte est la mise au propre d'une conférence donnée à la Freie Universität de Berlin en novembre 2014. Je tiens à exprimer ma gratitude à Joseph Ziegler (Université d'Haïfa) qui a bien voulu relire une première version de cet article. Mes remerciements vont également à Luca Bianchi (Université de Milan) et à Christophe Grellard (École pratique des Hautes Études, Paris) pour leurs suggestions et commentaires.
}

Irene Caiazzo, CNRS, PSL, Laboratoire d’Études sur les Monothéismes (UMR 8584)

D Open Access. (C) 2020 Irene Caiazzo, published by De Gruyter. (c) BY-NC-ND This work is licensed under a Creative Commons Attribution-NonCommercial-NoDerivatives 4.0 International License. 
1260, qui tout de suite fait l'objet de plusieurs « volgarizzamenti ». Très rapidement, certains sujets philosophiques paraissent donc sortir des salles de cours et avoir un écho plus large dans des milieux « laïques » ou, si l'on préfère, de non-spécialistes ; de toute évidence, les questions de la félicité et de la noblesse de l'homme ne concernent pas que les magistri artium mais tous les hommes, du moins ceux des classes supérieures. Un autre cas bien connu est celui de Jacques de Pistoia qui adresse sa Quaestio de felicitate au poète Guido Cavalcanti. Enfin, comme l'écrit Dante dans le Convivio « è manifesto che nobilitade umana non sia altro che seme di felicitade, messo da Dio ne l'anima ben posta, cioè lo cui corpo è d'ogni parte disposto perfettamente ${ }^{1}{ }^{1}$. Quelques lignes plus haut Dante a en effet expliqué que l'âme peut être mal à l'aise dans un corps qui ne possède pas une complexion équilibrée ${ }^{2}$. Si les connaissances médicales que ces remarques révèlent ont déjà été mises en avant par les spécialistes ${ }^{3}$, l'arrière-plan physiognomonique a en revanche été quelque peu négligé.

La physiognomonie, la science qui permet de connaître le caractère d'un homme (ou d'un animal) d'après son aspect physique, renaît en Occident au XII ${ }^{\mathrm{e}}$ siècle mais c'est à partir du XIII ${ }^{\mathrm{e}}$ siècle qu'on peut parler de véritable diffusion. Entre médecine, psychologie, astrologie et éthique, la physiognomonie tente d'expliquer l'inexplicable, à savoir le lien entre le corporel et l'incorporel, la relation d'interdépendance entre le corps et l'âme. Jole Agrimi a consacré diverses études fondamentales à la physiognomonie médiévale; au sujet du commentaire de Guillaume d'Aragon (ou d'Espagne) sur la Physiognomonie pseudo-aristotélicienne, dont il sera longuement question dans les pages qui suivent, elle écrivait que le but ultime de la physiognomonie rejoignait l'idéal de la philosophie en tant que recherche du vrai et de la béatitude terrestre et que le problème de la véritable noblesse de l'homme y était également posét . Pourtant, ces pistes de recherche

1 Dante Alighieri, Opere, vol. II : Convivio IV, xx, 9, éd. G. Fioravanti, Milan 2014, 714.

2 Ibid. IV, xx, 8, 713-714 : « Puote adunque l'anima stare non bene nella persona per manco di complessione, o forse per manco di temporale : ed in questa cotale questo raggio divino mai non risplende ». Le « temporale » renvoie aux saisons et aux mouvements des astres ; cf. ibid. IV, ii, 5-7, $550 ; 7$ : « E così la nostra mente, in quanto ella è fondata sopra la complessione del corpo, che [ha] a seguitare la circulazione del cielo, altrimenti è disposta uno tempo e altrimenti un altro ».

3 Je me contenterai de citer deux articles récents : A. Raffi, « Dante e l'embriologia aristotelica : il problema dell'origine dell'anima dal Convivio alla Commedia », Campi immaginabili 46-47 (2012), 5-38 ; P. Ureni, « Medicine and Radical Thought, a Possible Galenic Presence in the Commedia ", dans M. L. Ardizzone (éd.), Dante and Heterodoxy : The Temptations of 13th Century Radical Thought, Cambridge 2014, 225-241.

4 J. Agrimi, « La fisiognomica e l'insegnamento universitario : la ricezione del testo pseudoaristotelico nella Facoltà delle Arti » dans Ead., Ingeniosa scientia nature. Studi sulla fisiognomica 
n’ont pas trouvé preneur, les travaux de Jole Agrimi étant utilisés surtout par les historiens de la « philosophie de pourceaux », pour reprendre la célèbre expression de Pierre Duhem. Il m’a paru alors intéressant de regarder de près les textes de physiognomonie en circulation au XIII ${ }^{\mathrm{e}}$ siècle, pour la plupart encore inédits, afin d'y repérer un portrait physique et morale du philosophe, une physionomie du philosophe en quelque sorte. Alors que le portrait physique du philosophe est esquissé par le médecin et philosophe perse Razi, le portrait intellectuel et moral du philosophe (ou de l'homme noble) est élaboré par les auteurs latins du XIII ${ }^{\mathrm{e}}$ siècle en mettant bout à bout plusieurs passages des œuvres d'Aristote et du Centiloquium pseudo-ptoléméen.

\section{Le portrait du philosophe et la diffusion de la physiognomonie en Occident}

Une description physique du philosophe se lit dans le Kitāb al-Manșūrī de Razi, traduit en latin par Gérard de Crémone (avant 1187, année de la mort du traducteur) :

Signa hominis philosophi. Huius signa sunt, quod eius statura est erecta. Carnis quoque tenet aequalitatem et est albus parvam habens rubedinem admixtam. Capilli eius inter multos et paucos et planos et crispos sunt medii et inter nigros et rubeos. Manus quoque sunt planae digitos habentes divisos. Frons etiam est magna et oculi inter varios et nigros sunt medii, molles quoque sunt, et quod eius intuitus ridenti vel gaudenti similis invenitur ${ }^{5}$.

medievale, Florence 2002, 129-130 : « Il fine della fisiognomica si connette così all'ideale della filosofia come desiderio del vero bene e beatitudine terrena. È il valore del problema sollevato, l'essenza della nobiltà dell'uomo, individuata non nell'ereditarietà e nel lignaggio, ma nella nobiltà delle operazioni e delle azioni che gli sono più proprie in quanto uomo (la conoscenza del vero, la ricerca del bene, l'esercizio della virtù), che mostra con piena evidenza l'utilità e la necessità della fisiognomica, [...]. Di quella nobiltà, che riguarda principalmente l'anima, quell'intelletto di origine divina di cui aveva parlato Aristotele nel De generatione animalium, ma i cui segni si manifestano già nel corpo, nella perfetta qualità e disposizione delle parti ». Sur la félicité et le rôle du philosophe dans le commentaire de Guillaume de Mirica sur la Physiognomonie pseudo-aristotélicienne, cf. Ead. « Fisiognomica : nature allo specchio ovvero luce e ombre », dans Ead., Ingeniosa scientia nature, 73-79.

5 Razi, Liber Almansoris II, 51, dans Scriptores physiognomonici graeci et latini, éd. R. Förster, Leipzig 1893, vol. II, 175-176. L'édition Förster reproduit le texte de l'édition princeps publiée à Milan en 1481, qui à son tour correspond à la traduction latine de Gérard de Crémone. 
La doctrine physiognomonique exposée dans le livre II du Kitāb al-Manșūrī a son fondement dans la théorie des complexions des corps. Le mélange des quatre qualités - chaud, froid, sec et humide - détermine une complexion possédant des caractéristiques données ${ }^{6}$. À une complexion équilibrée, bien balancée, correspond un caractère plus parfait, car tempéré, qu'il sera possible de reconnaître justement à partir des signes physiques et de l'allure générale du corps. À la fin du livre II nous lisons la typologie des différents caractères humains. Cela va sans dire que la complexion du philosophe est bien balancée, c'est-à-dire que, pour chaque partie du corps prise en examen, il est au milieu entre deux extrêmes. Il y a d'autres chapitres qui sont également intéressants pour notre propos ; par exemple le chapitre 50 : «Signa corporis habentis bonam naturam ${ }^{7}$, et le chapitre 49 : « Signa hominis boni intellectus et bonae naturae ${ }^{8}$. Le « déterminisme » de la doctrine physiognomonique de Razi sera désamorcé par les lecteurs latins : j’y reviendrai plus loin. La traduction de Gérard de Crémone, souvent intitulée dans les manuscrits Liber Almansoris, connaît une large diffusion à partir du XIII ${ }^{\mathrm{e}}$ siècle ; le livre II circule aussi séparément du reste, comme s'il s'agissait d'une œuvre à part entière, avec pour titre Physionomia 9 . Mais sans aucun doute le fait le plus saillant est que le Liber Almansoris est traduit en langue vernaculaire, ce qui prouve son succès dans des milieux laïcs. Le livre II est utilisé dans le Régime du corps ou Livre de Physique du médecin Aldebrandin de Sienne, le premier traité diététique en langue vernaculaire (d'après Marilyn Nicoud), composé avant $1257^{10}$. Danielle Jacquart avait montré qu'il existe une traduction latine anonyme antérieure (dénommée version A) qui a servi de base à la traduction de Gérard de Crémone (version B) ${ }^{\mathbf{1 1}}$. Or, les recherches récentes de Rosa Piro, sur lesquelles je reviendrai plus loin, ont montré que le Régime du corps est en fait une adaptation en français de la version A du Liber Almansoris. La quatrième partie du Régime du corps est intitulée «Phisanomie »; Aldebrandin de Sienne promet au lecteur de lui apprendre

\footnotetext{
6 Sur le livre II, voir J. Agrimi, « Fisiognomica e 'Scolastica' », dans Ead., Ingeniosa scientia nature, 20 ; Ead., « Fisiognomica tra tradizione naturalistica e sapere medico », Ibid., 50-51 ; Ead., « La fisiognomica e l'insegnamento universitario », 104-105.

7 Razi, Liber Almansoris II, 50, 175.

8 Ibid. II, 49, 174-175.

9 Agrimi, « Fisiognomica e 'Scolastica' », 20.

$10 \mathrm{Cf}$. M. Nicoud, Les régimes de santé au moyen âge. Naissance et diffusion d'une écriture médicale (XIII $-X V^{e}$ siècle), I, Rome 2007, 114-144, 699. Livre de Physique est le titre qui se lit dans les manuscrits, comme l'écrit Marilyn Nicoud.

11 Le manuscrit de Paris, BnF, lat. 6906 a été étudié par Danielle Jacquart et par Rosa Piro à sa suite ; cf. D. Jacquart, « Note sur la traduction latine du Kitāb al-Manșūrī de Rhazès ", Revue d'histoire des textes 24 (1994), 359-374, et le livre de Rosa Piro cité plus loin à la note 15.
} 
à connaître les quatre tempéraments - sanguin, colérique, flegmatique, mélancolique - ainsi que les complexions de chaque membre du corps et, enfin, de reconnaître le tempérament de chaque homme à partir de ses membres ${ }^{12}$. Il faut noter que la galerie de types humains y fait défaut. Mais le déterminisme du Liber Almansoris est modéré par Aldenbradin dès le chapitre introductif ; les natures, surtout les mauvaises, peuvent être changées et corrigées grâce aux bons enseignements des hommes sages :

Et jasoit ce que selonc nature il doive ensi estre com nous vous dirons, il puet bien estre autrement, et ce puet estre par les bons enseignemens et por le doctrine que li homme retienent, car vous devés savoir que norreture tolt aucunes fois nature de ses drois par divers usages, si com vous veés tous jors en hommes et en bestes. En hommes poés veoir quant maint homme sont de si male nature que par leur nature ne doivent faire se mal non, et par les ensegnemens et por le doctrine des sages hommes, devienent bon et font autre cose que lor nature n'aporte ${ }^{13}$.

Dans le chapitre conclusif du livre II, Razi explique qu'il ne faut pas s'arrêter à un seul signe physique mais qu'il faut en prendre en compte plusieurs et les mettre ensemble, afin d'avoir une vision globale de la personne ; néanmoins les plus importants ce sont les signes du visage, tout particulièrement les yeux. Cette remarque pourrait faire l'écho au De complexionibus, où Galien met en garde contre les physiognomonistes et la démarche consistant à prendre en compte un seul élément de l'aspect extérieur du patient ${ }^{14}$. La Phisanomie d'Aldebrandin de Sienne reprend ce dernier chapitre du livre II du Liber Almansoris, avec pour titre «Du darerin capitele ».

En 1300 Zucchero Bencivenni traduit en florentin la totalité du Liber Almansoris, à partir de la version de Gérard de Crémone. L'Almansore comporte le chapitre sur le philosophe, dont la traduction est très fidèle au latin :

12 Le régime du corps de maître Aldebrandin de Sienne : texte français du XIII siècle, publié pour la 1re fois, d'après les manuscrits de la Bibliothèque nationale et de la Bibliothèque de l'Arsenal, éds. L. Landouzy et R. Pépin, Paris 1911, 193 : « C'est li commencemens comment on puet connoistre le nature de cascun homme par dehors, et se complexion. Pour ce que doné vous avons enseignemens en .ij. partíes que dit vous avons devant, comment vous poés conoistre les .iiij. complexions si com le sanguine, le colorike, le fleumatique, le melancolique et le complexion de cascun menbre de cors [et les complexions qui lor sont covignables et qui pueent metre le cors à nient], si vous laisserons à dire de ceste matere, et vous dirons enseignemens comment vous porrés connoistre le nature de cascun par les menbres que on voit dehors ».

13 Le régime du corps de maître Aldebrandin de Sienne, 193.

14 Galien, De complexionibus 2.6, dans Burgundio of Pisa's Translation of Galen's " De Complexionibus », éd. R.J. Durling, Berlin 1976 (Galenus Latinus I), 84. Cf. J. Ziegler, « Philosophers and Physicians on the Scientific Validity of Latin Physiognomy, 1200-1500 », Early Science and Medicine 12 (2007), 285-312. 
Capitolo .Lj. De' segni de l'huomo filosopho.

I cui segni ke la sua statura è diricta, e à 'qualitade di carne, et è biancho, e àe un poco di rosseza mescolata ; e i suoi chapelli intra poki e molti, e piani e crespi, sono mezani, e intra neri e rossi ; e le mani sono piane e ànno le dita divise ; e la fronte è grande ; e li occhi intra vai e neri son mezani e sono molli ; e ke 'l suo riguardo si truova somilliate a rridente e a llieto e gaudente $\mathrm{e}^{15}$.

Dans l'Almansore le chapitre 50 est intitulé « De' segni di conoscere l'uomo di buona natura ${ }^{16}$, et le chapitre 49 : "De' segni de l'homo k'è di ratto ingengno ${ }^{17}$. En outre, le chapitre conclusif du livre II a été interpolé avec des passages qui semblent provenir du chapitre initial du Régime du corps d'Aldebrandin de Sienne ${ }^{18}$. Donc, nous retrouvons les remarques visant à nuancer le déterminisme de Razi : la mauvaise nature d'un homme peut être modifiée avec l'enseignement et l'éducation. De toute évidence, Zucchero Bencivenni tout comme Aldebrandin de Sienne se soucie de nuancer le déterminisme excessif de la physiognomonie de Razi, l'aspect extérieur n'étant qu'un signe.

Rappelons brièvement que c'est à partir du XII ${ }^{\mathrm{e}}$ siècle que cette discipline fait sa réapparition dans l'Occident latin ${ }^{19}$. D'abord, avec l'ouvrage de l'Anonyme Latin, rédigé probablement au IV siècle, et fondé sur la Physiognomonie pseudo-aristotélicienne et sur Polémon ${ }^{20}$. Des copies manuscrites de l'Anonyme Latin ont été réalisées au XII ${ }^{\mathrm{e}}$ siècle ; il est cependant difficile de parler de véritable diffusion du texte à cette époque, qui est très rarement citée ${ }^{21}$. Le Secrets des secrets pseudo-aristotélicien contient une section sur la physiognomonie, dont la traduction latine de Philippe de Tripoli, des années 1230, circule à la cour de l'empereur Frédéric II ${ }^{22}$. Le premier traité de physiognomonie original a été composé

$15 \mathrm{Il}$ « volgarizzamento » a été édité pour la première fois par R. Piro, L’Almansore. Volgarizzamento fiorentino del XIV secolo, Florence 2011, 149.

16 L'Almansore, 148.

17 Ibid., 147-148.

18 Que l'Almansore de Bencivenni a été interpolé avec l'œuvre du médecin siennois est un fait avéré, comme nous en informe Rosa Piro.

19 Agrimi, « Fisiognomica : nature allo specchio », 63 ; Ead., « Fisiognomica tra tradizione e sapere medico ».

20 Anonyme Latin, Traité de physiognomie, texte établi, traduit et commenté par J. André, Paris 1981. 21 Cf. J. Ziegler, "The Biology of the Virtues in Medieval and Early Renaissance Theology and Physiognomy », dans M. Gadebusch Bondio et A. Bettels (éds.), Im Korsett der Tugenden. Moral und Geschlecht im kulturhistorischen Kontext, Hildesheim-Zürich-New York 2013, 13.

22 Les sections du Secretum Secretorum portant sur la physiognomonie ont été éditées par R. Förster, Scriptores physiognomonici graeci et latini, vol. II, 183-222. Voir l'article de M. Grignaschi, "La diffusion du Secretum secretorum (Sirr-al-'asr) dans l'Europe occidentale », Archives d'histoire doctrinale et littéraire au Moyen Âge 47 (1980), 7-70, et, en dernier lieu, S. J. Williams, « The Early Circulation of the Secret of Secrets », Micrologus 2 (1994), 127-144. La version courte du 
par Michel Scot, et constitue la troisième partie du Liber particularis, rédigé à l'intention de Fréderic II dans les années 1230. Michel Scot donne plusieurs définitions de la physiognomonie, dont la plus complète est celle-ci : « Phisionomia est la science ingénieuse de la nature par laquelle sont connus la vertu et le vice de tout animal ${ }^{23}$. Comme l'avait justement indiqué Jole Agrimi, la typologie des caractères humains fait défaut tant chez l'Anonyme Latin que chez Michel Scot ${ }^{24}$. À propos de ce dernier, Danielle Jacquart avait parlé de « sous-représentation de la psychologie ${ }^{25}$.

Les caractères humains sont en revanche présents dans la Physiognomonie pseudo-aristotélicienne, traduite du grec par Barthélemy de Messine, à la cour du roi Manfred dans les années 1260. On y chercherait en vain le portrait du philosophe, en revanche nous avons les « bene nati ${ }^{26}$, syntagme qui peut renvoyer à une catégorie humaine, l'intellectuel, et/ou sociale, l'aristocrate. J'y reviendrai plus loin. L'incipit fameux de la version latine de Barthélemy de Messine, qui suscita de longs excursus chez les commentateurs, est : " Quoniam et animae sequuntur corpora et ipsae secundum se ipsas non sunt impassibiles a motibus corporis ${ }^{27}$. Dans le prologue de la seconde partie de sa Phisonomia, Michel Scot rappelle l'un des axiomes de la physiognomonie, qui paraît s'appuyer sur le début de la Physiognomonie pseudo-aristotélicienne : « ... dicimus quod anime sequuntur corpus et ipse anime non sunt impassibiles idest sunt passibiles a motibus corporis in quibus morantur certo spatio vite temporalis ${ }^{28}$. Il y a un problème patent dans la chronologie : Michel Scot ne peut pas avoir consulté la traduction de Barthélemy de Messine. Une version latine antérieure a-t-elle existé ? Pour l'instant, nous n'avons pas de témoignage ou d'information qui puissent le

Secret des Secrets, traduit en latin par Jean de Séville dans les années 1130, connue sous le titre de Epistola Aristotelis ad Alexandrum, ne comporte pas la section sur la pysiognomonie. Cf. S. J. Williams, "Secret of Secrets ». The Scholarly Career of a Pseudo-Aristotelian Text in the Latin Middle Ages, Ann Arbor 2003.

23 Traduction de D. Jacquart, «La physiognomonie à l'époque de Frédéric II », Micrologus 2 (1994), 20, note 3 : « Phisionomia est ingeniosa scientia nature per quam cognoscuntur virtus et vitium cuiuslibet animalis ». Sur la finalité de la physiognomonie selon Michel Scot, voir aussi Agrimi, « Fisiognomica e 'Scolastica' », 20-27; Ead., « Fisiognomica : nature allo specchio », 62.

24 Agrimi, «La fisiognomica e l’insegnamento universitario », 103.

25 Jacquart, «La physiognomonie à l'époque de Frédéric II », 24.

26 Ps.-Aristoteles, Physiognomonica, I, 15, dans Scriptores physiognomonici graeci et latini, éd. Förster, vol. I, 29 : « Bene nati signa ». Sur le « bene natus », voir plus loin, 155-156.

27 Ibid., I, 1, 5.

28 Agrimi, « Fisiognomica e 'Scolastica' », 23. Ces correspondances avaient déjà été remarquées par R. Förster, Dissertatio de translatione latina Physiognomonicorum quae feruntur Aristotelis, Kiel 1884, 22-23. Cf. aussi Agrimi, « La fisiognomica e l'insegnamento universitario », 108. 
confirmer, et Michel Scot n'est pas non plus connu pour sa connaissance de la langue grecque.

L'éclosion de la physiognomonie en Occident paraît liée à la cour de Sicile, dans un cadre « laïque » donc, car il s'agit d'une discipline très utile aux hommes politiques qui doivent s'entourer de collaborateurs, savoir reconnaître les ennemis, bien choisir leurs conjoints afin de s'assurer d'une bonne descendance. La physiognomonie nait et se développe, du moins dans un premier temps, hors l'institution universitaire. À la différence d'autres sciences importées des arabes, telles que l'optique ou l'alchimie, elle n'était pas incluse dans le De divisione philosophiae de Dominique Gundissalvi, qui a eu une grande influence sur les classifications des sciences du XIII ${ }^{\mathrm{e}}$ siècle. Elle n'est pas mentionnée dans les guides des étudiants et dans les divisions de la philosophie ressortissants de la Faculté des Arts, éditées par Claude Lafleur. Avec et grâce à la traduction de Barthélemy de Messine, la Physiognomonie pseudo-aristotélicienne est insérée dans les manuscrits contenant les œuvres d'Aristote ; de ce fait, elle a fait l'objet de quelques commentaires universitaires et d'une progressive institutionnalisation, dont les étapes ont été étudiées dans le détail par Jole Agrimi. Une possible référence à la physiognomonie pourrait se trouver dans la liste des 219 erreurs condamnés par l'évêque de Paris, Etienne Tempier, en 1277. La bien connue proposition 207 dénonce le déterminisme astral et paraît faire l'écho du début de la Physiognomonie pseudo-aristotélicienne, puisque l'on affirme que l'âme suit le corps :

À l'heure de la génération d'un homme, en vertu de l'ordre des causes supérieures et inférieures, une disposition inclinant l'homme vers telles actions et tels événements est introduite dans son corps et, par conséquent, dans son âme qui suit le corps. - C’est une erreur, sauf si on l'entend relativement aux événements naturels, et par voie de disposition ${ }^{29}$.

La physiognomonie est mentionnée dans les statuts de l'université de Bologne de $1405^{30}$. Jean de Jandun la situe entre la philosophie naturelle et l'éthique, dans le prologue qu'il met en tête à ses Questions sur la Physique d'Aristote ${ }^{31}$; Buridan a également commenté la Physiognomonie pseudo-aristotélicienne. Quoi qu'il en

29 La condamnation parisienne de 1277. Nouvelle édition du texte latin, traduction, introduction et commentaire par David Piché, Paris 1999, 142 : «Quod, in hora generationis hominis, in corpore suo et per consequens in anima, que sequitur corpus, ex ordine causarum superiorum et inferiorum, inest homini dispositio inclinans ad tales actiones et eventus. - Error, nisi intelligatur de eventibus naturalibus, et per viam dispositionis ». Une relation entre la proposition 207 et la physiognomonie avait déjà été suggérée par Agrimi, « La fisiognomica e l'insegnamento universitario », 102, n. 5. J'aurais tendance à être plus affirmative que Jole Agrimi.

30 Agrimi, « Fisiognomica e 'Scolastica' », 11.

31 Ibid., 9 : «Et est illa scientia multum conveniens ad moralem scientiam et ad vitam practicam, quia cognoscere mores hominum naturales, quod docetur ibidem, multum confert ad 
soit, la physiognomonie ne quitta jamais véritablement les cours et son public laïque ; elle bénéficia d'une double vie en quelque sorte : à la cour c'est son application pratique et divinatoire qui l'importe, à l'université c'est une science théorique qui occupe une place dans la philosophie d'Aristote, en faisant la liaison entre plusieurs de ses parties. Cependant, il serait faux d'en conclure pour autant qu'il y a eu une physiognomonie de cour et une physiognomonie savante.

\section{Le commentaire de Guillaume d'Aragon}

L'un des premiers commentaires sur la Physiognomonie pseudo-aristotélicienne est celui de Guillaume d'Aragon (ou d'Espagne), conservé dans 18 manuscrits, et édité pour la première fois en 2006 par Paula Val Naval ${ }^{32}$. La forme de commentaire employée, du type sententia cum quaestionibus, est surtout utilisée à la Faculté des Arts, à partir des années 1240, selon Olga Weijers ${ }^{33}$. Le commentaire comporte une divisio textus et des lectiones : il a sans aucun doute été rédigé pour l'enseignement. Il est attribué à Guillelmus Hispanus en trois manuscrits ; mais il y a aussi une attribution fantaisiste à Petrus Hispanus ${ }^{34}$. Guillelmus Hispanus a été identifié avec Guillaume d'Aragon, l'auteur avéré du De nobilitate animi, qui a fait l'objet très récemment d'une nouvelle édition accompagnée d'une traduction anglaise ${ }^{35}$. Les lieux parallèles entre la Summa supra Phisonomiam et le De nobilitate animi mis en avant par Paula Val Naval semblent indiquer qu'il s'agit bien du même auteur ${ }^{36}$.

ordinatam conversationem cum hominibus et ad debitum regimen ipsorum hominum sicut sciunt experti in illis ».

32 P. Val Naval, Estudio, edición crítica, traducción y comentario de la «Summa supra Phisonomiam » de Guillermo de Aragón (c. 1300), Tesis doctoral, Universidad de Saragoza, 2006. Cette thèse m’a été signalée par Joseph Ziegler que je tiens à remercier ici.

33 O. Weijers, «La structure des commentaires philosophiques à la Faculté des Arts : quelques observations », dans G. Fioravanti, C. Leonardi et S. Perfetti (éds.), Il commento filosofico nell'Occidente latino (secoli XIII-XV), Turnhout 2002, 20 : « Dans ces commentaires, l'explication du texte, la sententia, est systématiquement combinée avec des questiones, des questions développées selon le schéma de base de la question disputée : formulation de la question, arguments pour l'une des deux réponses possibles, argument pour la position opposée, solution et réfutation des arguments contraires ».

34 Sur cette attribution à Pierre d'Espagne, cf. J. F. Meirinhos, «A atribuição a Petrus Yspanus das Sententie super libro de physonomia de Guillelmus Hispanus, no manuscrito Vaticano, Urb. lat. 1392 », Mediaevalia. Textos e Estudos 7-8 (1995), 329-359.

35 Guillelmus de Aragonia, De nobilitate animi, éd. et trad. W. D. Paden et M. Trovato, Cambridge (MA) 2013.

36 Val Naval, Estudio y comentario de la « Summa supra Phisonomiam », 36-37. 
Guillaume d'Aragon est également l'auteur d'un Liber de pronosticatione sompniorum $^{37}$, d'un commentaire inédit sur le Centiloquium pseudo-ptoléméen, et enfin d'un commentaire sur la Consolation de Philosophie de Boèce ${ }^{38}$. Très peu est connu de sa vie ; sans doute magister medicine à l'université de Montpellier (parfois on lit à Paris dans la littérature secondaire ${ }^{39}$ ). Il faut avouer que nous ne savons pas où le commentaire sur la Physiognomonie a été rédigé, bien que sa structure révèle sans ambages une visée pédagogique. Bornons-nous à quelques dates qui paraissent certaines, en mettant de côté les conjectures et les reconstructions hasardeuses. La rédaction du commentaire sur la Physiognomonie doit être située après 1260 , car Guillaume d'Aragon cite plusieurs traductions de Guillaume de Moerbeke (De generatione animalium, Rhetorica) et avant 1309, année mentionnée par le copiste dans le colophon du manuscrit conservé à la bibliothèque ambroisienne de Milan. De plus, le commentaire de Guillaume d'Aragon parait être employé dans la Compilatio phisionomiae de Pietro d’Abano, rédigée à Paris en 1295 ; mais l'emprunt en question, un seul, n'est pas très probant ${ }^{40}$. Le plus ancien manuscrit de la Summa supra Phisonomiam est le Paris, BnF, lat. 16089, un gros recueil composite des $\mathrm{XIII}^{\mathrm{e}}-\mathrm{XIV}^{\mathrm{e}}$ siècles, provenant du fonds de la Sorbonne, sur lequel plusieurs historiens se sont penchés ${ }^{41}$. Il contient, entre autres, la Compilatio phisionomiae de Pietro d'Abano, dédiée à un laïc, Bardellone Bonacolsi, capitaine général de la

37 R. A. Pack, «De pronosticatione sompniorum libellus Guillelmo de Aragonia adscriptus », Archives d'histoire doctrinale et littéraire du Moyen Âge 33 (1966), 237-293. Ce traité est rapidement analysé par A. de Libera, Penser au Moyen Âge, Paris 1991, 294-295, qui le situe vers 1330, après le Convivio de Dante donc. En fait, les œuvres de Guillaume d'Aragon doivent être situées en amont plutôt qu'en aval de Dante.

38 C. Olmedilla Herrero, Edición crítica de los comentarios de Guillermo de Aragón al « De consolatione Philosophiae » de Boecio, Tesis doctoral, Universidad Complutense, Madrid 1997. Je tiens à remercier chaleureusement Carmen Olmedilla Herrero de m’avoir envoyé son édition, dont la parution est prévue dans le « Corpus Christianorum », Brepols. Cf. aussi Ead., « Un accessus medieval : el comentario de Guillermo de Aragón al De consolatione Philosophiae de Boecio ", dans M. Pérez González (éd.), Actas del II Congreso nacional de latín medieval (León, 11-14 Noviembre de 1997), vol. 2, Léon 1999, 709-720.

39 Cf. P. Glorieux, La Faculté des Arts et ses maîtres au XIII ${ }^{e}$ siècle, Paris 1971, 163 : « n¹46, Guillaume d'Aragon », et $167:$ « n¹57, Guillaume d'Espagne ». Cf. O. Weijers, Le travail intellectuel à la Faculté des Arts de Paris : textes et maîtres (ca 1200-1500), Vol. 3 : Répertoire des noms commençant par G, Turnhout 1998, 101-103 (101) : « Guillelmus de Aragonia (prem. moitié du XIVe s.), médecin et astrologue, actif à Montpellier ou en Aragon ».

40 En revanche, Jole Agrimi et Danielle Jacquart écrivent que c'est Guillaume d'Aragon qui cite l'ouvrage de Pietro d'Abano.

41 Cf. D. Jacquart, « Autour de la Compilatio phisionomiae de Pietro d'Abano », dans Ead., Recherches médiévales sur la nature humaine. Essais sur la réflexion médicale (XII $-X V^{e}$ s.), Florence 2014, 355-371. Sur ce manuscrit, voir également E. Randi, «'Philosophie de pourceaux’ e re taumaturghi. Nota su un manoscritto parigino », Quaderni medievali 22 (1986), 129-137. 
ville de Mantoue, le commentaire de Gilles d'Orléans à l'Ethique à Nicomaque, le Sophisma de sensu agente de Jean de Jandun, les Questions de Jean Vath sur le De generatione animalium $^{42}$. Surtout, il faut signaler le De nobilitate animi de Guillaume d'Aragon, que les derniers éditeurs situent dans les années 1280-1290 ${ }^{43}$ - il s'agit du plus ancien témoin manuscrit conservé, qui a servi de base à l'édition. Enfin, dans sa préface à la traduction française de la Consolation de Philosophie de Boèce, intitulée Li Livres de Confort de Philosophie et dédiée à Philippe IV Le Bel, Jean de Meun (mort en 1305 ?) utilise - en fait il traduit mot à mot du latin - le prologue de Guillaume d'Aragon au commentaire sur la Consolation de Philosophie ${ }^{44}$. Toutes ces attributions mériteraient d'être examinées à nouveaux frais ; en tout cas, on peut raisonnablement situer l'activité de Guillaume d'Aragon dans le quatrième quart du XIII ${ }^{\mathrm{e}}$ siècle.

La longue préface par laquelle s'ouvre le commentaire sur la Physiognomo$n e^{45}$ a pour but de montrer l'utilité de la physiognomonie qui permet de repérer, à l'intérieur d'une société, les hommes qui sont naturellement disposés à réaliser des actions nobles ou, au contraire, des actions viles. Le ton est donné : la physiognomonie a une fonction éthico-politique. Le commentaire débute par une définition de la noblesse tirée de la Rhétorique d'Aristote dans la traduction de Guillaume de Moerbeke, selon laquelle la noblesse d'un peuple ou d'une ville se mesure d'après l'ancienneté de ses chefs politiques ${ }^{46}$. Une citation de la Poli-

42 L. Cova, "Le Questioni di Giovanni Vath sul De generatione animalium », Archives d'histoire doctrinale et littéraire au Moyen Âge 59 (1992), 175-287.

43 Guillelmus de Aragonia, De nobilitate animi, XIII. Cf. ibid. : «In De pronosticacione sompniorum he says he once spoke with 'a certain king' under circumstances that suggest that the king may have been Pedro III, who ruled Aragon from 1276 to 1285. No doubt he also served Alfonso III (1285-1291) and Jaime II (1291-1327). He may have been the beneficiary of an act by Alfonso, who in 1288 returned certain possessions that had been confiscated in a time of civil war to 'Master Guillelmus, our physician' ».

44 R. Crespo, «Il prologo alla traduzione della Consolatio Philosophiae di Jean de Meun e il commento di Guglielmo d'Aragona », dans W. den Boer, P. G. Van der Nat, C. M. J. Sicking et J. C. M. van Winden (éds.), Romanitas et christianitas. Studia Iano Henrico Waszink, A. D. VI Kal. Nov. A. MCMLXXIII, XIII lustra complenti oblata, Amsterdam-Londres 1973, 55-70. Lodi Nauta situe la rédaction du commentaire de Guillaume d'Aragon à la fin du XIII ${ }^{\mathrm{e}}$ siècle : cf. L. Nauta, «The Consolatio : the Latin commentary tradition, 800-1700 », dans J. Marenbon (éd.), The Cambridge Companion to Boethius, Cambridge 2009, 251-275.

45 L'édition de Val Naval comporte praefatio, alors que Jole Agrimi parle de lectio prima.

46 Val Naval, Estudio y comentario de la « Summa supra Phisonomiam », 119 : « Sicut dicit Philosophus primo sue rethorice nobilitas est genti et civitati sui iuris antiquos esse presides, in quo Philosophus optime innuit nobilitatis originem et formam qualitatis ipsius ». Cf. Aristoteles, Rhetorica I, 5 (translatio Guillelmus de Moerbeka), ed. B. Schneider, Leiden 1978 (AL XXXI/1-2), 175 : « Nobilitas quidem igitur est genti et civitati sui iuris antiquos esse, et presides primos in- 
tique d'Aristote sert ensuite à prouver que les hommes doivent vivre en société : l'homme est le meilleur et le plus parfait des animaux, mais il est le pire des animaux quand il est éloigné de la loi et de la justice ${ }^{47}$. Les philosophes utilisent la physiognomonie pour évaluer les hommes, puisque la bonne disposition d'un organe est le signe d'une bonne vertu organique, et les vertus organiques parfaitement disposées permettent une utilisation parfaite de l'intellect, de sorte que la vertu intellective ne soit pas entravée dans ses opérations les plus nobles. Vient ensuite une quaestio scolastique en bonne et due forme sur la noblesse - probablement la plus ancienne conservée ${ }^{48}$ : est-il plus noble celui qui est noble à cause de la seule noblesse de ses parents, ou celui qui est rendu noble par la bonté de ses actions ? Bref, c'est la question toujours débattue entre le noble de souche et le noble par ses mérites : «Primum est utrum nobilior sit qui sola nobilitate parentum est nobilis, aut qui bonitate operationum propriarum fit nobilis $»^{49}$. Plusieurs arguments à faveur et contre les deux thèses sont présentés selon le modèle de la question disputée ${ }^{50}$; la conclusion est qu'il est plus noble celui qui est noble à cause de la bonté de ses propres opérations. Le De nobilitate animi de

signes, et multos insignes prodiisse ex ipsis in hiis que zelantur ; singularis autem nobilitas que a viris aut mulieribus, et legitimitas ab ambobus, et, sicut in civitate, primos notos aut in virtute aut divitiis aut in alio eorum que honorantur, et multos insignes ex genere et viros et mulieres et iuvenes et senes ". La translatio anonyma, une traduction à partir du grec antérieure à celle de Guillaume de Moerbeke, est différente : cf. ibid., 21. La datation de la traduction de Guillaume de Moerbeke n'est pas connue avec précision ; elle aurait été réalisée peu avant 1269.

47 Val Naval, Estudio y comentario de la "Summa supra Phisonomiam », 121-123 : « Animadvertentes igitur philosophi quod homo animal est civile et quod in bono proprie operationis ita diversificatur et deficit, viderunt esse necessariam scientiam per quam possent de facili iudicare de quolibet, utrum sic esset naturaliter dispositus quod suas haberet operationes nobiles sive viles. Que 'phisonomia' dicitur, sic dicta a 'phisis' quod est 'natura' et 'noma' quod est 'nomen' quasi 'nominans naturam' vel naturales dispositiones, quibus possit argui quis qualem habeat naturali inclinatione in bonitate proprie operationis vel malitia qualitatem. Multum enim necessarium et utile est homini quod aliquam artem habeat per quam vilium et pravorum sciat vitare consortium et sciat societates eligere studiosas. Sicut enim dicit Philosophus primo Politicorum, sicut perfectum optimum animalium est homo, sic separatum a lege et iustitia pessimum omnium ».

48 Cette quaestio a échappée aux historiens de la philosophie médiévale qui se sont occupés de la noblesse, bien que Jole Agrimi l'avait signalée dans son article « La fisiognomica e l'insegnamento universitario : la ricezione del testo pseudoaristotelico nella Facoltà delle Arti ». Il faut également citer la dispute sur la noblesse, qui aurait eu lieu à la cour de Frédéric II, cf. F. Delle Donne, « Una disputa sulla nobiltà alla corte di Federico II di Svevia », Medioevo Romanzo 23/1 (1999), 3-20.

49 Val Naval, Estudio y comentario de la « Summa supra Phisonomiam », 127.

50 Voir, par exemple, ibid., 135 : « In oppositum arguitur [...]. Ad questionem dicendum videtur quod [...]. Ad primum igitur in oppositum dicendum quod, per interemptionem minoris et ad probationem, dico, $[. .]$.$» .$ 
Guillaume d'Aragon soutient le même point de vue ; ce qui n'est pas absolument original en soi. Par exemple, dans la Consolation de Philosophie, Boèce avait déjà défendu la noblesse fondée sur le mérite personnel ${ }^{51}$. Comme Jole Agrimi l'avait jadis indiqué, pour développer la thèse de la nova nobilitas, les deux ouvrages de Guillaume d'Aragon prennent comme point de départ le De bello Jugurtino de Salluste $^{52}$. Jusqu'à preuve du contraire, ce sont les deux seuls textes médiévaux qui s'appuient sur la définition de Salluste ${ }^{53}$. Suit alors une deuxième quaestio sur la noblesse : «Secundum quod queritur est [...] utrum speculativus nobilior sit activo ». En effet, après avoir démontré que c'est l'opération noble qui rend l'homme noble, Guillaume d'Aragon explique que, puisque dans l'homme il existe deux opérations nobles, c'est-à-dire l'active et la contemplative, il faut se demander si l'homme spéculatif est plus noble que l'homme actif. L'homme spéculatif est plus noble car à travers son opération il « s'assimile » (assimilatur) aux substances les plus nobles, comme le dit Aristote dans le livre $\mathrm{X}$ de l'Ethique à Nicomaque. La conclusion est qu'il faut donc préférer la félicité spéculative (felicitas speculativa) à l'active et que le spéculatif est plus noble $\mathrm{e}^{54}$. En prenant appui sur plusieurs autorités, dont Ghazali, Cicéron et Aristote, Guillaume d’Aragon affirme que la spéculation est le but de l'action ; nous nous conduisons d'une manière vertueuse pour que, au final, la connaissance de la vérité nous rende heureux ${ }^{55}$. Il n'y a pas de référence à la félicité ultra-terrestre ; Dieu n'est pas nommé et on ne sait pas très bien quelles sont les substances les plus nobles auxquelles l'homme spéculatif est censé s'assimiler ${ }^{56}$. Dans le livre X de l'Ethique - poursuit

51 Boèce, Consolatio Philosophiae III, m6.

52 Agrimi, « La fisiognomica e l'insegnamento universitario », 126, note 114.

53 Val Naval, Estudio y comentario de la « Summa supra Phisonomiam », 131 : «Quod recte intellexit Salustius in Iugurtino, qui, cum presentaret ei quidam ad confirmandum in senatorem, obiectum fuit illi quod non erat nobilis genere, erat tamen optimus in se, et tunc ait Salustius : 'Nova nobilitas michi est in quo melius est eam peperisse, quam ab aliis habitam corrupisse'. In quo expresse intelligit Salustius quod iste fit corruptor nobilitatis, qui parentes habuit nobiles et de se non est nobilis, alius vero genitor et conservator. Istud etiam valde patet per se. Quantumcumque enim quis habuit album patrem, si ipse fuerit niger in se, quis erit ausus dicere quod sit albus ? Certe nullus sane mentis. Concedo igitur rationes probantes hanc partem ».

54 Ibid., 135 : « [...] ille nobilior videtur qui per operationem suam nobilioribus substantiis assimilatur ; sed talis est speculativus, sicut dicit Philosophus decimo ethicorum. Unde ex hoc prefert felicitatem speculativam active, erit ergo speculativus nobilior ».

55 Ibid. : «Ex hoc igitur quod dictum est, quod speculatio addit supra actionem moralem, accipitur una ratio, quod speculativus sit nobilior ; alia, quia speculatio finis est actionis. Virtuose enim vivimus ut nos ultimo felicitet cognitio veritatis ».

56 Assimilatio n'est pas un terme d'Aristote ; il est employé dans la traduction latine du commentaire de Michel d'Efèse sur l'Ethique à Nicomaque. Cf. à ce propos, L. Bianchi, « Felicità intellettuale, 'ascetismo' e 'arabismo' : nota sul De summo bono di Boezio di Dacia », dans M. Bettetini 
Guillaume - Aristote affirme que la vie spéculative se suffit à elle-même et qu'elle est plus noble et meilleure que la vie active, car elle n'a pas besoin des biens extérieurs ; selon la vie spéculative l'homme est plus divin, selon la vie active il est plus humain ${ }^{57}$. La "félicité intellectuelle » est un thème débattu au XIII siècle, notamment à la suite de l'Ethique à Nicomaque qui fait l'objet de cours à la Faculté des Arts. Je ne citerai ici que Boèce de Dacie, auteur du De summo bono, qui exalte la vie spéculative, Siger de Brabant, Gilles d'Orléans, Thomas d'Aquin, Albert le Grand. Sur la base de ces courts passages, il est difficile d'évaluer la véritable place de Guillaume d'Aragon dans la discussion sur la « félicité intellectuelle », un sujet porteur de l'historiographie philosophique depuis une trentaine d'années ${ }^{58}$. Il me semble que Guillaume d'Aragon est un aristotélicien « pur » et qu'il ne pense pas que la vie spéculative culmine en Dieu - en tout cas il ne le dit pas de manière explicite ; la « félicité » qu'il vise se réalise non pas après la mort mais dans l'horizon de la vie humaine, dans la société civile, même si le spéculatif est un peu à l'écart des autres hommes, puisqu'il se suffit à lui-même. Chez Guillaume d'Aragon, les questions de la noblesse, du philosophe et de la félicité sont manifestement liées.

Après la préface, Guillaume d'Aragon en vient au commentaire du début du texte : "Quoniam et anime sequuntur corpora et ipse secundum se ipsas non sunt impassibiles a motibus corporis ». Tel que les autres commentateurs de la Physiognomonie pseudo-aristotélicienne, il se heurte à une difficulté majeure : le problème n'est pas tant que l'âme affecte le corps mais que le corps affecte l'âme qui est la forme du corps. Que le corps puisse affecter l'âme, cela est évident dans les cas de l'ivresse et des maladies, qui altèrent le spiritus ; ce qui, par conséquent, empêche l'âme de mouvoir convenablement le corps, puisque c'est bien à travers le spiritus que l'âme est le moteur du corps. Dans le cas de l'état amoureux, c'est à l'inverse le corps qui est affecté par l'âme, laquelle « movetur per speciem apprehensam ${ }^{59}$. Pour illustrer la relation entre l'âme et le corps, Guil-

et F. D. Paparella (éds.), Le felicità nel Medioevo, Louvain-la-Neuve 2005, 13-34 (sur l'assimilatio, en particulier, 31).

57 Val Naval, Estudio y comentario de la « Summa supra Phisonomiam », 135-137 : « ... secundum quod Philosophus accipit decimo ethicorum, quare Philosophus indicat magis per se sufficientem vitam speculativam quam activam et meliorem et nobiliorem. Non enim ita indiget bonis exterioribus, nec est ad aliud ordinata. Et secundum hanc magis divini, secundum aliam vero magis humani $»$.

58 Il serait impossible de mentionner ici toutes les contributions sur la félicité intellectuelle ; je me contenterai de citer le volume collectif Le felicità nel Medioevo (cf. note 56), et le récent article de L. Bianchi, « L'averroismo di Dante : qualche osservazione critica », Le Tre Corone. Rivista internazionale di studi su Dante, Petrarca e Boccaccio 2 (2015), 71-109 (surtout 93-109).

59 Val Naval, Estudio y comentario de la « Summa supra Phisonomiam », 143-145. 
laume d'Aragon fait appel à plusieurs auteurs (surtout Aristote) et expose toute sorte d'arguments qu'il détaille avec soin. Entre autres, celui de l'analogie avec l'artisan : l'âme suit le corps de la même façon que l'artisan « est dit » suivre son instrument. La cause (l'artisan) opère non seulement selon sa potentia mais aussi selon la faculté de son instrument ${ }^{60}$. C'est dans cette perspective que l'on peut affirmer que l'âme suit le corps.

La primauté de l'âme est également prônée par Averroès lorsqu'il affirme que les membres du cerf ne différent pas des membres du lion, si ce n'est à travers l'âme et ses puissances ${ }^{61}$; cet argument est évoqué à deux reprises par Guillaume d'Aragon $^{62}$. La citation provient du Grand Commentaire sur le De anima ${ }^{63}$, où Averroès veut démontrer l'absurdité de la thèse pythagoricienne de la transmigration des âmes, qu'Aristote a critiqué dans le De anima (livre I, 407b20-24). Guillaume d'Aragon ne fait pas allusion au contexte polémique ; la citation lui sert à prouver la relation étroite entre l'âme et le corps et la dépendance de ce dernier des vertus de l'âme qui est sa forme, son entéléchie. Ce même passage du Grand Commentaire est en revanche cité sans la mention d'Averroès (ou du Commentator) par Siger de Brabant dans son De anima intellectiva ${ }^{64}$, qui s'en sert lui-aussi pour critiquer les " fables pythagoriciennes ", d'après lesquelles n’importe quelle âme pourrait rentrer dans n'importe quel corps. Cet extrait du

60 Ibid., 153-155; cf. aussi 161-163.

61 Ibid., 323 : «In oppositum arguitur ex dicto Averrois, qui dicit quod membra cervi a membris leonis non differunt nisi quia anima differt ab anima, in quo expresse innuit quod ex eo quod anime sunt differentes, inter se et per potentias suas, corpora cervi et leonis sint diversificata, inter se et per membra sua. Dispositiones igitur tales in corpore erunt propter tales virtutes anime, et non e contrario ».

62 Ibid., 313 : « Concludit ex dictis duas conclusiones, et est prima quod virtutibus anime similes corporis forme fiunt, et vult idem dicere quod Commentator, qui dixit quod membra cervi a membris leonis non differunt nisi quia anima ab anima differt virtutibus et potentiis ».

63 Averroes Cordubensis, Commentum magnum in Aristotelis De anima libros 53, éd. F. S. Crawford, Cambridge (MA) 1953, 74-75 : « Videmus enim quod quidlibet habet formam propriam et corpus proprium, idest animam propriam et corpus animalis proprium. Et hoc quod dicit est manifestum in speciebus valde. Membra enim leonis non differunt a membris cervi nisi propter diversitatem anime cervi ab anima leonis. Et si esset possibile ut anima leonis existeret in corpore cervi, tunc Natura ociose ageret. Et hoc etiam manifestum est in individuis eiusdem speciei ; et ideo diversi sunt mores. Et ex hoc destruximus opinionem Pitagoricam ».

64 Sigerus de Brabantia, Tractatus de anima intellectiva, 2, éd. B. Bazan, Louvain 1972, 75 : « Et ideo male determinaverunt de anima nihil dicentes de susceptibili corpore, ac si quaelibet anima quodlibet corpus posset ingredi secundum pythagoricas fabulas. Nunc autem non est ita, immo secundum differentiam animarum, oportet esse et corporum susceptibilium differentiam, ita ut non differant membra leonis a membris cervi, nisi quia differt anima ab anima. Et hoc rationabiliter, quia forma non est nisi in propria materia ; anima autem est corporis forma, ut visum est ». 
Grand Commentaire est également inséré, dépourvu de son contexte, parmi les sentences du Commentator dans les Auctoritates Aristotelis ${ }^{65}$. Il faut noter que ni Albert le Grand ni Thomas d'Aquin ne citent ce passage du Grand Commentaire dans leurs commentaires respectifs sur le De anima I, 407b20-24. Mais dans la Quaestio de spiritualibus naturis - rédigée tout comme la Sentencia libri de anima à l'époque de la régence romaine (1265-1268) ${ }^{66}$ - Thomas d'Aquin cite le passage pour prouver la relation stricte entre la forme et la figure qui contient la forme, sans évoquer la critique des pythagoriciens ${ }^{67}$. La relation d'interdépendance entre le corps et l'âme est le présupposé même de la science physiognomonique ; ce qui s'accorde avec la théorie aristotélicienne selon laquelle l'âme est la forme d'un corps spécifique - « connaturaliter corpus et anima sunt cause ${ }^{68}$. La théorie platonicienne qui postule la préexistence des âmes et conçoit le corps comme la prison de l'âme ou encore la théorie pythagoricienne de la transmigration de l'âme sont difficilement compatibles avec la physiognomonie. De surcroît, Guillaume d’Aragon affirme que Démocrite et Pythagore ne suivent pas le principe du De caelo et mundo, selon lequel Dieu et la nature ne font rien en vain, puisqu'ils ont postulé beaucoup de choses inutiles, notamment la présence des âmes rationnelles dans les minéraux, les plantes et les animaux bruts ${ }^{69}$. Au final, après avoir débattu plusieurs questions, en présentant les arguments pour et contre, Guillaume d'Aragon établit que l'âme a la suprématie sur le corps : la

65 Auctoritates Aristotelis (De anima I - Commentator), dans Les Auctoritates Aristotelis. Un florilège médiéval. Étude historique et édition critique, éd. J. Hamesse, Louvain 1974, 176 : « <29> Membra leonis non differunt a membris cervi nisi propter diversitatem animae leonis ab anima cervi ».

66 Cf. la chronologie des œuvres de Thomas dans P. Porro, Tommaso d'Aquino. Un profilo storico-filosofico, Rome 2012.

67 Thomas de Aquino, Quaestio disputata de spiritualibus creaturis, art. 4, arg. 9, éd. J. Cos, o.p., Rome-Paris 2000, 50.

68 Val Naval, Estudio y comentario de la « Summa supra Phisonomiam », 149 : « Cum nichil aliud sit phisonomizare quam per dispositiones et figuras totius corporis et membrorum mores naturales et passiones in anima investigare, quarum connaturaliter corpus et anima sunt cause, sicut dictum est ; et dicitur etiam primo phisicorum hoc idem. Forma enim et materia causa omnium sunt ».

69 Ibid., 157-159 : «Sequitur enim ex quodam principio quo frequenter utitur Philosophus manifesto, scilicet quod in natura nichil sit frustra. Cum enim sit natura ars divina, sicut patet XI de animalibus, per Philosophum manifestum est quod cum deus nichil frustra faciat, quod etiam natura nichil facit frustra et hoc expresse dicit primo celi et mundi, quod deus et natura nichil faciunt frustra. Essent autem multa frustra si ita esset ut Democritus et Pyctagoras posuerunt. Frustrarentur enim anime rationales in mineralibus et plantis et brutis secundum nobiliores operationes suas, ex quo insipientia et impotentia argueretur divina, quod est valde irrationabile et absurdum ». 
configuration du corps et de ses membres sont de telle manière à cause de telles puissances de l'âme, et pas autrement : « dispositiones corporis et membrorum sunt tales vel tales propter potentias anime tales vel tales et non e contrario $»^{70}$. Les dispositions naturelles des corps sont données par la vertu informative au moment de la génération, laquelle, selon Aristote, est proportionnée aux mouvements des astres ; ce qui garantit en quelque sorte que ces dispositions ne soient pas accidentelles ou non-naturelles ${ }^{71}$. Les astres et la causalité astrale certifient la validité de la science physiognomonique. J’y reviendrai plus loin.

De toute évidence, les questions philosophiques suggérées par la Physiognomonie pseudo-aristotélicienne font l'objet de longs développements, tandis que les données physiognomoniques à proprement parler reçoivent moins d'attention. Guillaume d'Aragon explique qu'Aristote a d'abord « physonomisé » de manière historique, c'est-à-dire descriptive, et qu'il a ensuite expliqué les causes $^{72}$. Il s'agit de la même stratégie interprétative mise en œuvre par les commentateurs des traités biologiques d'Aristote (auxquels Guillaume fait de nombreux renvois) : l'Histoire des animaux décrit les animaux, alors que la Génération des animaux expose les causes ${ }^{73}$. La galerie de caractères humains est ainsi analysée de manière hâtive. Par exemple, les bene nati ${ }^{74}$ sont ceux qui sont bene complexionati $^{75}$, que l'on peut rapprocher de ce qu'Albert le Grand a écrit dans

70 Ibid., 323 : «Consequenter queritur utrum membrorum dispositiones sint tales vel tales, scilicet talis complexionis vel figurationis vel compositionis, propter tales vel tales virtutes anime vel potentias, aut e contrario tales vel tales sint in anima potentie vel virtutes propter talem vel talem complexionem vel compositionem in membris ». Sur ces questions, voir Ziegler « Philosophers and Physicians on Latin Physiognomy ", 297-298.

71 Val Naval, Estudio y comentario de la «Summa supra Phisonomiam », 325 : « Ad questionem istam breviter dicendum quod dispositiones corporis et membrorum sunt tales vel tales propter potentias anime tales vel tales et non e contrario, sicut optime concludunt rationes ad partem istam. Intelligo tamen de dispositionibus 'naturalibus' corporis et membrorum, quas a virtute informativa in sui generatione acquirit, que movet ad proportionem celi, sicut dicit Philosophus XVI de animalibus, et quo de huiusmodi dispositionibus querit non de innaturalibus vel de hiis que a causis per accidens possunt introduci ».

72 Ibid., 309 : « Postquam docuit Philosophus phisonomizare historice non tangendo causas, in parte ista docet phisonomizare considerando causas vel ea ex quibus cause sufficienter habentur ».

73 Pour une vue d'ensemble, voir l'article très général de S. Perfetti, «I libri De animalibus di Aristotele e i saperi sugli animali nel XIII secolo », dans C. Crisciani, R. Lambertini et R. Martorelli Vico (éds.), «Parva naturalia ». Saperi medievali, natura e vita, Atti dell’XI Convegno della Società Italiana per lo Studio del Pensiero Medievale (Macerata, 7-9 dicembre 2001), Pise-Rome 2004, 143-170.

74 Ps.-Aristoteles, Physiognomonica, I, 15, 29-31 : «Bene nati signa ».

75 Val Naval, Estudio y comentario de la « Summa supra Phisonomiam », 281-283. 
son commentaire sur les Topiques, à savoir que les bene nati ont des esprits (spiritus) subtils, des opérations animales aisées, jointes à une bonne raison ${ }^{76}$. En tout cas, Guillaume d'Aragon n'associe pas le bene natus avec le philosophe ou avec l'homme noble, qu'il s'agisse du noble par lignage ou par ses propres opérations. C'est dans un autre passage qu'il revient sur le " philosophe » et la description physique qu'en donne le Liber Almansoris. Les signes physiques du philosophe type, dont Razi parle, sont valables aussi pour un philosophe particulier, et ils permettent donc de reconnaître une attitude, c'est-à-dire à une disposition naturelle à devenir homme de science. Les propos de Razi sont confirmés par Ptolémée dans la première proposition de son Centiloquium :

Preterea Rasis in phisonomia sua dat signa philosophi ; quare, secundum ipsum, si philosophi dantur signa phisonomie, etiam et particularis philosophi possunt dari. Preterea hoc idem potest argui ratione, accipiendo hoc manifestum : quod nullus efficitur artifex vel sciens in aliqua facultate, qui ad hoc non est dispositus aptitudine naturali. Sed dispositus habet signa phisonomizata, ergo per signa phisonomizata contingit cognoscere artifices vel scientes. Maior huius rationis, licet sit plana, confirmari potest per Ptholomeum in prima propositione Centilogii et per commentatorem Haly. Dicit enim Ptholomeus quod scientia astrorum ex te et ex illis est, hoc est, ex aptitudine naturali ad hoc in te et ex motibus et naturis astrorum, et eadem ratione de aliis scientiis est verum. Minor plana est de $\mathrm{se}^{77}$.

Le mariage entre la physiognomonie et l'astrologie est consommé. La citation provient de la traduction arabo-latine de Platon de Tivoli du Centiloquium pseudo-ptoléméen, la plus répandue au Moyen Âge : «Verbum primum. Scientia astrorum ex te et illis est. Astrologus non debet dicere rem specialiter sed universaliter... » ; Haly est l'auteur fictif d'un commentaire à lemmes, lui-aussi

76 Albert le Grand, Super libros octo Topicorum 8, éd. A. Borgnet, t. II, Paris 1890, 521 : « Quia qui nati sunt bene, hoc est, quibus bene innatum est, hoc possunt bene facere : quia ingenium dicitur intus genitus, et hic est qui subtiles habet spiritus et expeditas animales operationes, et bonam rationem collativam ». Sur le bene natus, voir A. A. Robiglio, « The Thinker as a Noble Man (bene natus) and Preliminary Remarks on the Medieval Concepts of Nobility », Vivarium 44, 2-3 (2006), 205-247 ; pour une discussion sur ce passage d'Albert le Grand, cf. 230. Sur les Topiques en particulier, voir ibid., 226-227, 229. Cf. aussi le De sompniis de Boèce de Dacie : «Et quia virtus naturaliter inclinatur in suum bonum et suum delectabile, ex hoc contingit, quod quidam homines contemplativi bene nati ad scientias ex parte corporis sui et animae nec impediti ab eis propter curas exteriores incipiunt profunde perscrutari de eo quod cogitant admirantes ob defectum causae, sicut quidam nuper vehementer admirantes, quomodo homini per somnium potest fieri praecognitio eventuum futurorum, de quibus numquam cogitavit, rogabant instanter, ut eis scriberem, quid per somnium sciri possit et quomodo ", cité d'après Robiglio, 235-236. Cf. aussi A. A. Robiglio, « Dante bene nato : Guido Cavalcanti e Margherita Porete in Par. V, 115 », L'Alighieri. Rassegna dantesca 26 (2005), 45-62.

77 Val Naval, Estudio y comentario de la « Summa supra Phisonomiam », 243-245. 
amplement diffusé, sur le Centiloquium, qu'il faudrait identifier avec Abū Ja'far Aḥmad ibn Yūsuf ibn al-Dāya ${ }^{78}$. Voici la transcription du commentaire d'Haly sur le premier verbum :

Quod dixit Ptholomeus ex te et illis significat quod qui res futuras scire desiderat duabus viis oportet intendere. Una ut aspiciat motus stellarum et opera que fiunt in rebus secundum motum earum, et libros quos antiqui scripserunt de significatione motuum ipsarum, et adiungat hiis quecumque probavit in suo tempore ; ideo dixit ex illis. Alia via est quando a sibi per divinam inspiracionem consideraverit istam scientiam multa vera poterit dare iudicia. Multi enim sunt qui dant vera iudicia quorum nec significationes nec rationes invenimus in operatore si vero in operato, sed quomodo dicant ex eorum cordibus manat, et ex his multos vidimus et haec via, cum pura fuerit, a philosophis divina dicit. Ideo dixit Ptholomeus quod eorum qui sunt sub circulo lune, quidam habent huius artis scientiam per doctrinam, alii vero per inspirationem divinam ${ }^{79}$.

Guillaume d’Aragon a également commenté le Centiloquium ; trois manuscrits sont connus de ce texte inédit qui a pour incipit : "Sicut dixit Ptholomeus in proverbiis Almagesti. Non fuit mortuus... ${ }^{80}$. Il débute par un prologue qui fait l'éloge de la science et de l'astrologie, en établissant que tous les événements d'ici bas sont régis par les mouvements nécessaires des corps célestes :

Sicut dixit Ptholomeus in proverbiis Almagesti : non fuit mortuus qui sapienciam vivificavit, nec fuit pauper qui intellectui dominatus est. [...] ostendit maxime homines vivere est opus proprium videlicet opus intellective virtutis cui proprie et per se debetur, scientie vel sapientie inventio et recordacio. Hiis enim duobus vivificatur, sapientia scilicet invenienda non inventa et exercitando sopita. Hoc est quod Ptholomeus intendit quantum ad partem primam suis proverbii ${ }^{81}$. [...] Alio modo considerantur celestia secundum quod per suas virtutes habent movere et continue et esse principia motiva omnium motuum qui sunt hic. Sicut enim dicit philosophus primo Metheorum, iste motus continuus est illi ex necessitate ut enim omnis gubernetur ${ }^{82}[\ldots]$.

78 Sur les différentes versions du Centiloquium voir, en dernier lieu, J.-P. Boudet, « Nature et contre-nature dans l'astrologie médiévale. Le cas du Centiloquium du pseudo-Ptolémée ", dans M. van der Lugt (éd.), La nature comme source de la morale au Moyen Âge, Florence 2014, 383-410.

79 Paris, BnF, lat. 7480, f. 1v-2r.

80 Val Naval, Estudio y comentario de la « Summa supra Phisonomiam », 70-71. Il en existe également une traduction française inédite dans un manuscrit du XIV siècle, conservé à la Bibliothèque nationale de France, fr. 1349, f. 245 : « La Sentence sus centiloge de PTHOLOME, faite par maistre GUILLAUME D’ARRAGON », avec pour incipit : «Si come dit Ptholomé ou proverbe del Almages : Cil ne fu pas mort... ».

81 Paris, BnF, lat. 7480, f. 3v.

82 Paris, BnF, lat. 7480, f. 5r. 
Quant à la première proposition du Centiloquium, elle fait l'objet d'un long développement, dont voici quelques courts extraits, en particulier celui où Razi est expressément cité, qui fait le pendant du commentaire sur la Phisiognomo$n e^{83}$ (par ailleurs, une étude serrée des deux textes serait fort souhaitable) :

\begin{abstract}
Dicit ergo quod astrorum scientia est ex te. Et hoc tripliciter : aut quantum ad prudentem applicationem, aut quantum ad naturalem proprietatem et inclinationem, aut quantum ad divinam infusionem ${ }^{84}$. [...] Et tales fuerunt illi qui per se principes scientiarum fuerunt. Talis fuit Ypocras in medicina, Aristoteles in naturalibus, Ptholomeus in astronomia. Talem creationem animarum videretur intelligere Rasys et alii astrologi, quando Mars, Venus et virtus pervidentur secundum exigenciam sciencie stellarum in signis, hec est naturalis et divina infusio et prima de qua loquitur hic Ptholomeus et Haly que fit mediantibus superioribus... ${ }^{85}$.
\end{abstract}

Revenons maintenant au commentaire sur la Physiognomonie. Guillaume d'Aragon explicite ce qu'il faut entendre par aptitude naturelle à la science. Il distingue ainsi trois degrés chez les hommes : les premiers possèdent une aptitude naturelle qui les pousse à l'acte, de la sorte qu'ils passent facilement, par eux-mêmes, des choses sensibles aux choses universelles qui sont le début de l'art ou de la science. Ces hommes-là sont les inventeurs des sciences ${ }^{86}$. Ensuite, viennent les hommes qui ont besoin d'un enseignant et qui, avec un peu d'aide et sans trop de travail, apprennent et s'accomplissent. Enfin, ceux pour qui est nécessaire un enseignant et beaucoup de travail. Peu d'hommes du premier degré se rencontrent, plusieurs du second et du troisième. Dans chacun des trois types, on décèle une aptitude naturelle à la science, mais à des degrés différents ${ }^{87}$. La

83 La Phisiognomonie est ouvertement citée et attribuée à Aristote : cf. Paris, BnF, lat. 7480, f. 14 r.

84 Paris, BnF, lat. 7480, f. 7r.

85 Paris, BnF, lat. 7480, f. $7 \mathrm{v}$.

86 Val Naval, Estudio y comentario de la « Summa supra Phisonomiam », 245 : « Cum igitur habitus artium et scientiarum sint forme quedam et actus informantes ipsos artifices et scientes, planum est quod, si aliquis debet esse artifex vel sciens in aliquo, quod oportet eum habere aptitudinem naturalem. Est tamen advertendum quod ista aptitudo in hominibus tripliciter invenitur. Quidam enim ita sunt apti quod quasi per se moventur ad actum, et de facili ex motibus sensibilium excercitantur ad universalia, que sunt principium artis vel scientie, ad quam habent inclinationem naturalem ; et tales fuerunt inventores scientiarum ».

87 Ibid., 245-247 : " Alii vero sunt qui ab istis cadunt et faciunt gradum secundum, qui ad hoc, ut perficiantur, indigent doctore. Sunt tamen in tantum apti quod auxilio doctoris sine magno studio perficiunt et addiscunt. Alii vero sunt qui, cum auxilio doctoris, adhuc eos multum laborare oportet, multo tamen labore perficiuntur in doctrina. In primo gradu pauci inveniuntur ; in secundo vero et tertio plures. In omnibus tamen est naturalis aptitudo, licet secundum magis et minus ». 
conclusion est que, à travers les signes physionomiques, il est possible de reconnaître chez un homme l'aptitude naturelle à la science et non pas un homme de science en acte, car l'aptitude naturelle peut être entravée dans son actualisation. Beaucoup d'hommes aptes aux sciences, intelligents, ne sont pas dans la réalité des hommes de science accomplis. C'est pour cette raison qu'Aristote affirme qu'il est impossible de reconnaître un médecin ou un joueur de cithare à travers la physiognomonie. En revanche, il admet qu'il est possible de détecter une aptitude naturelle ; en effet, les philosophes se servent de la physiognomonie pour évaluer les enfants dans la société, et comprendre lequel est apte à une telle science ou à un tel art, afin qu'ils soient éduqués par la communauté ou par les amis de la façon la plus appropriée ${ }^{88}$.

La méritocratie naturelle et le projet pédagogique prônés par Guillaume d'Aragon forcent notre admiration. C'est parce qu'il met en avant le rôle de l'enseignant et la possibilité donc de développer une prédisposition naturelle chez l'homme grâce à l'éducation qu'il se démarque du déterminisme de la physiognomonie ancienne et de Razi. Mais qui « forme » donc cette élite intellectuelle ? Quelle est la cause de cette aptitude naturelle à la science ? Ce sont les astres, nous l'avions déjà vu. C'est à travers la vertu des corps supérieurs et incorruptibles que le monde inférieur est régi, comme l'affirme Aristote dans le premier livre des Météores et surtout dans le XVI livre Des Animaux (en fait il s'agit du De generatione animalium II, 3), lorsqu'il dit que la vertu informative est proportionnée à la configuration des mouvements des astres. Il s'ensuit nécessairement que les corps de ce monde sont formés, forgés et gouvernés selon les différents configurations et mouvements des astres ${ }^{89}$ qui sont les causes de la physionomie ou de la figure du corps humain, telles qu'ont été expérimentées de manière astrologique par les anciens philosophes. D’abord, il sera question de l'influence des douze signes du Zodiaque sur l'aspect du corps humain et, ensuite, de celle des sept planètes :

88 Ibid., 247 : « Unde ad questionem, cum queritur utrum passiones artium (vel artifices vel scientes) cognoscamus vel possimus cognoscere per signa phisonomizata, dico quod non secundum actum, sed secundum aptidudinem naturalem sic, que multipliciter impediri potest in homine ne reducatur ad actum. Unde multos invenimus aptos ad scientias, bene intelligentes et subtiles, qui artifices non sunt facti. Cum ergo Philosophus hic intendat dare artem phisonomizandi per signa concludentia actum primum in anima talis vel talis passionis, propter hoc dicit quod per phisonomiam non contingit cognoscere medicum vel citharistam. Non tamen negat quin aptum natum possumus cognoscere. Immo, in rei veritate, philosophi ad hoc utebantur phisonomia, iudicando pueros civitatis, quis eorum ad quas artes vel scientias esset aptus et in illis instituebantur a communi vel ab amicis ».

89 La référence au premier livre des Météores se trouve également dans le commentaire de Guillaume d'Aragon sur le Centiloquium, cf. supra, 157. 
Cum in virtute superiorum et incorruptibilium corporum iste mundus inferior gubernetur, sicut dicit Philosophus primo metheororum et XVI de animalibus dicens quod virtus informativa proportionata est coordinationi astrorum motibus ${ }^{90}$, sequitur necessario quod secundum diversas ordinationes astrorum diversimode moveantur, informentur et figurentur et gubernentur corpora huius mundi. Ut igitur perfecte habeamus causas phisonomie vel figurationis corporis humani, super ea que naturaliter sunt accepta superius, hic accipienda videntur que astrologice ab antiquis philosophis experta : primo, quas figuras prebeant vel ad quas moveant signorum virtutes ; secundo, de planetis dicetur hoc idem ${ }^{91}$.

C'est ainsi que s'achève le commentaire de Guillaume d'Aragon : en fait il s'agit de la clé de voute de sa théorie physiognomonique et de sa philosophie toute entière. Les astres régissent le monde d'ici-bas ; la causalité astrale explique tous les phénomènes, y compris la nature individuelle de chaque homme, sa configuration physique et son attitude éventuelle à la science. La Physiognomonie pseudo-aristotélicienne n'avait pas son fondement dans la causalité astrale ; il s'agit là d'une innovation herméneutique toute médiévale. Le passage du $D e$ generatione animalium est également mis à contribution dans le De nobilitate animi pour valider la thèse de la noblesse par mérite ; le Centiloquium et la Physiognomonie sont aussi cités ${ }^{92}$. La pensée de Guillaume d'Aragon est cohérente.

90 Aristote, De generatione animalium II, 3 (translatio Guillelmi de Moerbeka), éd. H.J. Drossaart Lulofs, Paris-Bruges 1966, 54 : « Relinquitur autem intellectum solum deforis advenire et divinum esse solum : nichil enim ipsius operationi communicat corporalis operatio. Omnis quidem igitur anime virtus altero corpore visa est participare, et diviniore vocatis elementis : ut autem differunt honorabilitate anime et vilitate invicem, sic et talis differt natura ; omnium quidem enim in spermate inexistit quod facit gonima esse spermata, vocatum calidum. Hoc autem non ignis neque talis virtus est, sed interceptus in spermate et in spumoso spiritus aliquis et in spiritu natura, proportionalis existens astrorum ordinationi ». La référence aux astres ne se lit pas dans la traduction arabo-latine, intitulée De animalibus, de Michel Scot ; ce dernier avait de toute évidence à sa disposition un manuscrit arabe avec un texte défectueux. Je remercie mon collègue Marc Geoffroy (CNRS, Paris) de son expertise éclairé sur le texte arabe. Sur les différences entre les traductions et l'interprétation de ce passage de $G A$ par Pietro d'Abano et Thomas d'Aquin, voir maintenant D. Jacquart, « La complexion selon Pietro d'Abano », dans Recherches médiévales sur la nature humaine, 373-416 (surtout 406-409).

91 Val Naval, Estudio y comentario de la « Summa supra Phisonomiam », 377.

92 Guillelmus de Aragonia, De nobilitate animi, 1, 11, 20-23, 86-88 : « [...] sed quia celum uniformiter non potest se habere in formatione omnium corporum, oportet quod difformiter informetur. Unde De animalibus dicit quod virtus informativa proportionaliter operatur coordinationi astrorum. Anima igitur, que naturalem habet inclinationem ad corpus et ipso in suis operationibus utitur tamquam organo vel obiecto necessario, inducitur ad agendum per ipsum secundum aptitudinem corporis ipsius ; et istam talem inclinationem significant astronomi per stellas, et naturales vel phisionomi per membrorum figuras. Aliter non est intelligendum quod Philosophus dicit in Physonomia et Ptholomeus in Centilogio ». 


\section{Quelques remarques conclusives}

L’astrologie est la reine des sciences ; la physiognomonie a une validité car elle a comme fondement la causalité astrale. C'est une véritable cosmologie que Guillaume d'Aragon met en place, en s'appuyant sur la philosophie naturelle d'Aristote et l'astrologie arabe. Albert le Grand avait lui-aussi subordonné la physiognomonie à l'astrologie. Dans la Compilatio phisionomiae, Pietro d'Abano explique que hyleg et alchocoden - dans l'astrologie médiévale le donneur de vie et celui qui détermine la durée de la vie - transmettent le pouvoir des corps célestes, les causes universelles premières, aux deux causes de la formation de l'embryon : la virtus informativa qu'il appelle aussi l'intellect ou la partie divine et qui joue le rôle à la fois de l'artisan et de la forme, et le spiritus contenu dans le sperme qui est à la fois l'instrument et la matière ${ }^{93}$. Pietro d'Abano expose ensuite la théorie de la double chaleur : la chaleur non ignée contenue dans le spiritus du sperme, qui provient des astres, et la chaleur élémentaire ignée sont la cause de la vie et de la formation de l'embryon ${ }^{94}$. Cette théorie prend appui sur le De genera-

93 Pietro d'Abano, Compilatio phisionomiae, BnF, lat. 16089, f. 111ra : «Quorum unum ut multum locum tenet organi et materie, et hic spiritus quidem existit in spumoso spermate interceptus ; alterum vero factoris et forme virtus videlicet informativam intellectus se ures divina vocatus ». Le texte a été collationné avec l'édition princeps, Padoue 1474. Voir l'étude de Jacquart « Autour de la Compilatio phisionomiae de Pietro d'Abano »; E. Paschetto, « La fisiognomica nell'enciclopedia delle scienze di Pietro d'Abano », Medioevo 11 (1985), 97-111, surtout 105-107. Sur la Compilatio, voir aussi G. Federici Vescovini, « La simmetria del corpo umano nella Physiognomica di Pietro d'Abano : un canone estetico », dans G. Piaia (éd.), « Concordia discors ». Studi offerti a Giovanni Santinello, Padoue 1993, 347-360.

94 Pietro d'Abano, Compilatio phisionomiae, BnF, lat. 16089, f. 111ra : «Et iste quidem spiritus primitus complantatus seu cognatum callidum spumoso semini immixtus, dupplici calore est affectus. Quorum alterum vivificum et celeste, naturam habet proportionalem astrorum ordinationi, et ipsum peripathethice scole Aristoteles princeps posuit a corpore separabile. Eius enim actio ex instrumentorum non fit aliquo corporali. Reliquum vero est calorem elementare igneum consumptum per quod dictum est ab Aristotele nullum animal generari. Si itaque res divina intelligentia vocata fuerit spiritu substentante prefato duplici calore ut determinatis utitur organis, altero ipsius non corporeo principali, quo intellectus vocati operatio astrorum operationi secundum quid redditur similis, donec partium principaliori facta vitam demum susceperit propriam. Quare et gratiose dictum fuit hic, scilicet vocatum intellectus quod est virtus informativa, principium fore a corpore separatum, cum eius actus non perficiatur organo corporali. Operatur enim hic dictus intellectus, practici cuiusdam operatione intellectus, quod quidem est ut organo iam dicto medio sperma gonimum reddatur in vitam animam omnem susceptio impinguat, ut et formam debitam ex humido cuilibet partium inducatur [...] ». Le texte a été collationné avec l'édition princeps, Padoue 1474, qui est très fautive. 
tione animalium II, 3, dans la version de Guillaume de Moerbeke ${ }^{95}$. La corrélation entre les astres, les signes zodiacaux et le développement de l'embryon était déjà évoquée dans un traité physiognomonique anonyme et, d'une manière plus générale, Michel Scot avait lui-aussi relié l'âme et l'embryon aux cours des corps célestes ${ }^{96}$. Comme Pietro d'Abano, Guillaume d'Aragon cite le passage du De generatione animalium II, 3, - la vertu formative est proportionnée à la configuration des mouvements des astres - en se montrant en cela très au fait de ce qui était à la mode parmi ses contemporains. Ce même passage justifie sa théorie de la noblesse par mérite dans le De nobilitate animi et dans le Liber de pronosticatione sompniorum ${ }^{97}$.

Revenons à notre point de départ : est-ce que Dante a eu connaissance de la quaestio sur la noblesse et la félicité spéculative contenue dans le commentaire de Guillaume d’Aragon sur la Physiognomonie pseudo-aristotélicienne ? Est-ce qu'il a connu la théorie à la fois biologique, médicale et astrologique de la formation et de l'animation de l'embryon telle qu'elle est exposée dans la Compilatio phisionomiae de Pietro d'Abano ? Nous ne pouvons pas le jurer, puisque, à la différence de Pétrarque et de Boccace, nous n'avons pas les manuscrits de Dante et nous ne savons pas non plus quelle bibliothèque il a réellement fréquentée ${ }^{98}$. Rappelons que, bien qu'il ne se soit pas penché sur la Compilatio phisionomiae,

95 Cf. le texte cité à la note 90. Sur le De generatione animalium II, 3 au XIII ${ }^{\mathrm{e}}$ siècle, cf. les articles de B. Nardi (qui pourtant ne parle pas de la Compilatio phisionomiae) réunis dans Id., Studi di filosofia medievale, Rome 1960 : «L'origine dell'anima umana secondo Dante » et « L'anima umana secondo Sigieri ». Sur Pietro d'Abano, voir toujours de B. Nardi, « La teoria dell'anima e la generazione delle forme secondo Pietro d'Abano », dans Id., Studi sull'aristotelismo padovano dal XIV al XVI secolo, Firenze 1958, 1-17, et en dernier lieu Jacquart, « La complexion selon Pietro d'Abano ». Sur la virtus informativa et l'animation de l'embryon, cf. M. Van der Lugt, Le ver, le démon et la vierge. Les théories médiévales de la génération extraordinaire, Paris 2004, 79-89. Sur Aristote, cf. G. Freudenthal, Aristotle's Theory of Material Substance : Heat and Pneuma, Form and Soul, Oxford 1992, et maintenant C. Cerami, Génération et Substance. Aristote et Averroès entre physique et métaphysique, Berlin 2015, ch. IV : « La mise en œuvre de l'étude de la génération substantielle : la génération animale ».

96 Cf. R. A. Pack, « Auctoris incerti de physiognomonia libellus », Archives d'histoire doctrinale et littéraire du Moyen Âge 41 (1974), 113-138. Sur Michel Scot, cf. Agrimi, « Fisiognomica e 'Scolastica' », 23.

97 Pack, «De pronosticatione sompniorum libellus », 261 : «Et gratia huius virtutis quam corpus sequitur dicit Philosophus sexto decimo De animalibus quod omnis anima diviniore quodam corpore participare videtur vocatis elementis. Quamvis enim corpus materialiter sit ex quattuor elementis, non tamen per elementa ad hoc deducitur ut aptum fiat ad talis vel talis anime informationem, sed ad hoc aptatur per virtutem corporis celestis. Unde Philosophus ibidem dicit quod virtus que corporis est formativa, proportionata est astrorum virtutibus ».

98 Cf. l'article de synthèse de R. Zanni, « Una ricognizione per la biblioteca di Dante in margine ad alcuni contributi recenti », Critica del testo 17/2 (2014), 161-204. Cf. S. Gentili et S. Piron, « La 
Bruno Nardi avait déjà rapproché Dante de Pietro d'Abano, non pas pour « découvrir une source empirique » mais pour « comprendre la pensée de Dante dans sa véritable signification historique ${ }^{99}$. Quoi qu'il en soit des sources avérées ou rêvées, l'homme noble du IVème livre du Convivio n'est pas né par génération spontanée $^{100}$. Les débats philosophiques que je me suis efforcée d'éclairer dans ces pages, qu'ils soient issus ou pas des magistri artium, en constituent l'indispensable toile de fond.

bibliothèque de Santa Croce ", dans J. Chandelier et A. Robert (éds.), Frontières des savoirs en Italie à l'époque des premières universités (XIII ${ }^{e}-X V^{e}$ siècles), Rome 2015, 481-507.

99 B. Nardi, « Dante e Pietro d'Abano », Nuovo giornale Dantesco 4/1-2 (1920), 1-15, réimpr. dans Id., Saggi di Filosofia Dantesca, Milan-Naples 1930, 43-65. Je traduis de l'italien certains propos des pages 43-44.

100 Alain de Libera a écrit de très belles pages sur l'homme noble chez Dante, cf. Penser au Moyen Âge, 268-298. Cependant, je ne partage pas l'interprétation selon laquelle l'intellectuel laïque, l'homme noble, de Dante serait une nouveauté sans précédent, en rupture avec la tradition universitaire. 
